\title{
Learning stochastic finite-state transducer to predict individual patient outcomes
}

\author{
Patricia Ordoñez $^{1} \cdot$ Nelson Schwarz ${ }^{1}$ - Adnel Figueroa-Jiménez ${ }^{2}$. \\ Leonardo A. Garcia-Lebron ${ }^{3}$. Abiel Roche-Lima ${ }^{4}$
}

Received: 30 August 2016 / Accepted: 5 October 2016 / Published online: 17 October 2016

(C) The Author(s) 2016. This article is published with open access at Springerlink.com

\begin{abstract}
The high frequency data in intensive care unit is flashed on a screen for a few seconds and never used again. However, this data can be used by machine learning and data mining techniques to predict patient outcomes. Learning finite-state transducers (FSTs) have been widely used in problems where sequences need to be manipulated and insertions, deletions and substitutions need to be modeled. In this paper, we learned the edit distance costs of a symbolic univariate time series representation through a stochastic finite-state transducer to predict patient outcomes in intensive care units. The Nearest-Neighbor method with these learned costs was used to classify the patient status within an hour after $10 \mathrm{~h}$ of data. Several experiments were developed to estimate the parameters that better fit the model regarding the prediction metrics. Our best results are compared with published works,
\end{abstract}

This article is part of the topical collection on Health and Technology in Latin America

Patricia Ordoñez

patricia.ordonez@upr.edu

Abiel Roche-Lima

abiel.roche@upr.edu

1 Rio Piedras Campus, University of Puerto Rico, San Juan, Puerto Rico

2 Interamericana University in Fajardo, Fajardo, Puerto Rico

3 University College of San Juan, San Juan, Puerto Rico

4 Medical Sciences Campus, University of Puerto Rico, San Juan, Puerto Rico where most of the metrics (i.e., Accuracy, Precision and Fmeasure) were improved.

Keywords Prediction of patient outcomes · Machine learning Classification and visualization of physiological data

\section{Introduction}

Current methods for measuring the well-being of a patient in the intensive care unit (ICU) acquire a patient's vital signs data at rates that are difficult for a human to analyze $(60-500 \mathrm{~Hz})$. These measurements are displayed on a monitor for a few seconds as a collection of univariate time series and then lost to further analysis. Instead, a lower-frequency version of this data is stored in an electronic health record after validation by a medical provider at the rate of once every $15 \mathrm{~min}$ to once every several hours. Physicians make life-saving decision based on this lower-frequency data. Recently, however, there has been interest in storing and analyzing the high-frequency data using automated and semiautomated methods [1]. Many are recognizing the importance of analyzing this data as a multivariate temporal representation by creating multivariate probabilistic models [2] or temporal abstractions [3] from electronic health records or creating multivariate structures that are similar to those in other domains such as convolutional neural networks [4] or imaging [5]. 
In related work, four multivariate time series representations were examined to serve as compressed representation of high frequency physiological data from Intensive Care Units: Stacked Bags-of-Patterns, Multivariate Bags-of-Patterns, Multivariate Piecewise Dynamic Time Warping and Ensemble Voting with Bag-of-Patterns [6]. The representations were tested in three distinct data domains: field-motion capture data, robot sensor data, and ICU data. Two data sets were examined in each domain for a total of six different data sets. Similarity was measured by converting the data sets into the indicated representations and then classifying the data using the Nearest Neighbor algorithm. The results demonstrated that the multivariate representations outperformed univariate ones for the purpose of predicting the targeted outcome.

This paper represents work in the univariate time series domain which we are examining for application to the multivariate domain. We are using Stochastic Edit Distance on a concatenated symbolic representation of the time series to classify a physiological data set for an acute episode of hypotension. In this case, the edit cost probabilities are learned by a stochastic Finite-State Transducer (FST) [7]. We compare the best results in the multivariate domain to the results we achieved in the univariate domain with this new approach to test its potential effectiveness.

\section{Methods}

\subsection{Dataset description}

To compare results, we used one of the data sets from previous work [6] which came from the Physionet Challenge [8,9]. It consists of 1-6 days of highfrequency physiological data from patients in an Intensive Care Unit (ICU). The prediction task was to classify which patients were going to enter an episode of acute hypotension in the forecast window of one hour after the last entry in the data set. We focused only on heart rate for this trail and instead of using leave one out validation, we used the test set that was provided in the Challenge and the original data from previous work as the training set.

The training set consisted of 58 of 60 patients from the 2009 Physionet Challenge, of which 28 experience an episode of hypotension in the hour following the period of the data sample. The data was taken from segments A and B which contained all the hours of data prior to the period in which the patient may or may not have experienced an episode of hypotension. Two patients were dropped because of large gaps in the training data resulting in the final size of 58 . The test set consisted of segment B of Test Set A which contained 10 patients, of which half had experienced a period of acute hypotension following the sample data, and was composed of only $10 \mathrm{~h}$ of data.

\subsection{Data representation}

The data for this paper was converted to a symbolic representation named Symbolic Aggregate approXimation (SAX) [10] which is explained in more detail in the Methods section. This representation normalizes the data in overlapping local windows prior to conversion such that the mean is 0 and standard deviation is 1 . In some windows the variation in values was 0 which resulted in values that divided by zero. To correct for this, any subsequence that had zero variation was substituted by values of 0 .

The second factor that required a difference in procedure for extracting the data was a matter of computational limitation. Our stochastic transducer, at the moment of this study, could not run on strings of length longer than 1020 characters. Consequently, the desired string lengths were achieved by using dimensionality reduction in the SAX algorithm. We reduced each string by counting any run of equal values as one value (i.e., $a b b$ abb abb aaa abb would be reduced to abb aaa aab, as abb is only counted once when repeated).

If this reduction was insufficient to achieve the desired length, all subsequences were joined into a single vector and we extracted the data using systemic random sampling. This sampling technique involves the selection of $\mathrm{n}$ elements from an ordered sampling frame of $\mathrm{N}$ elements, every $\mathrm{k}$ times. Systemic sampling was under the assumption that it best preserves any patterns along the time series data that was converted. A simple random sample may have, for example, involved more data from the earlier times of the time series, instead of equally across the sample.

If the single vector containing all the data has a length of $10,000(N)$, and the desired length is 1000 $(n)$, the $\mathrm{k}$ would be $10(\mathrm{~N} / \mathrm{n})$. Every 10th element would be sampled. However, this assumes every single letter in our character string is independent from the rest. Instead, our SAX strings are grouped because they originate from time series subsequences. We sampled, not 
every $\mathrm{k}$ letter, but every $\mathrm{k}$ group of letters. The size of the group is determined by the size of alphabet parameter in the SAX algorithm. Following our previous example, if the vector is formed by groups of size 5 (i.e. using a, b, c, d, and e), our k would be every $50((\mathrm{~N} / \mathrm{n})$

$*($ size of alphabet $)=10 * 5)$. If every 50th element is sampled, the final sample would be 200 groups of 5 characters, hence a string of 1000 characters. Such sampling resulted in a decrease in the training set between 5 and $20 \%$ of the original time series.

After the original vector of subsequences is reduced to a desired string length, it is concatenated to form a single string per time series. Each time series corresponds to a patient who either did or did not undergo an episode of hypotension. Patients who did undergo an episode of hypotension are classified as 1 , while other that did not is labeled 0 . Depending on the original time series classification, the final SAX string was attached to its appropriate label. The resulting output from our time series to SAX conversion was, for example, " 1 abbaccabcaba..." or "0 abaacbabcaaa..."

\subsection{Algorithms}

Before applying the learning Finite-Transducer for Stochastic Edit Distance to the time series data, the data was converted into the Symbolic Aggregate approXimation (SAX) representation [10]. This representation reduces the dimensionality of a time series by converting it into a collection of symbolic representations. The algorithm uses three variables: WindowSize, the number of values that can be represented by a SAX word; Symbols, the number of symbols in a SAX word (the bins on which the values are averaged for the representation); and finally Alphabet, the number of characters in a SAX word (the number of letters that can be used in the word to describe low to high values of distribution). Each SAX word represents the same amount of data and is calculated using an overlapping sliding window to capture all patterns in the data. These representations are a collection of words for each time series.

\subsubsection{Definitions}

Edit distance is widely used to compute similarities between pairs of strings. It is defined as the minimum number of operations, i.e. insertions, deletions, and substitutions, required to transform the input into the output. Stochastic edit distance is defined when the costs of the operations become random variables because the transformations are based on arbitrary phenomena. It can be modeled as a stochastic transduction, compiled in the form of a 2-tape automaton. This model is called stochastic finite-state transducer. It has resulted being very useful for sequence problems, such as pattern recognition, segmentation, DNA alignment and sequence classifications [7, 11, 12].

\subsubsection{Learning stochastic edit distance}

Figure 1a represents a finite-state transducer (FST), also called memoryless transducer, which allows one to compute edit distance using the pre-defined costs. Figure $1 \mathrm{~b}$ represents a FST where the costs can be learned from a training set as the probability of each operation, i.e. Stochastic Finite State
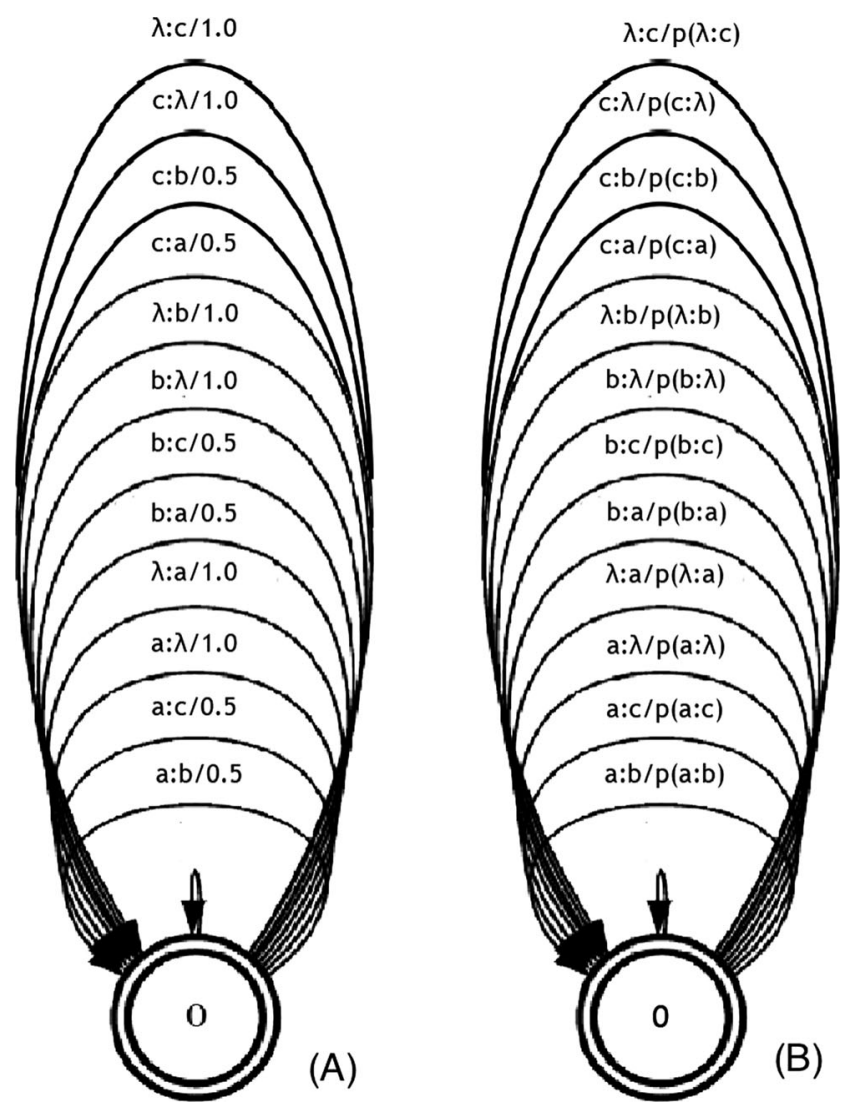

Fig. 1 Finite-state transducer with alphabet $[a ; b ; c ; \lambda]$, where $\lambda$ represent the empty symbol. a deterministic - with pre-define costs $\mathbf{b}$ stochastic costs as probabilities 
Transducers. In our case, an unbiased learning algorithm (Algorithm 1) using a stochastic conditional finite-state trans- ducer was implemented to learn the probabilities associated with the edit distance costs.

\begin{abstract}
Algorithm 1 Unbiased Learning of a Conditional Edit Distance Transducer
Learning stage

Step 1: The Learning Set $\left(L S_{i}\right)$, represents the $i$-th sequence in the training set. Each sequence was classified as $1=$ positive to a hypotension scenario within an hour or $0=$ negative to a hypotension scenario within an hour.

Step 2: From each $L S_{i}$, a set of sequence pairs (pairdataset) $P S_{i}$ was built in the form $(x ; N N(x))$, $\forall x \in L S_{i}$, where $N N(x)=\operatorname{argmin}_{y \in L S_{i}-\{x\}} d_{E}(x, y) \quad\left(d_{E}\right.$ is the classic edit distance and $N N$ is the Nearest Neighbor).

Step 3: A unique conditional finite-state transducer $t$ was learned from $\cup_{i} P S_{i}$ (which represents all pair of sequences included in pairdataset $P S_{i}$ )
\end{abstract}

\title{
Validation stage
}

For each sequence $x^{\prime}$ in the Test Set $T S$, i.e. each $x^{\prime} \in \cup_{j} T S_{j}$ (which represents each sequence in the test set):

Step 4: for each sequence $y_{i}$ in the learning dataset, $y_{i} \in \cup_{i} L S_{i}$, the conditional probability, $p\left(y_{i} \mid x^{\prime}\right)$, was computed, using the conditional transducer $t$ (where $t$ represents the learned edit cost probabilities).

Step 5: $x^{\prime}$ was classified as the same class as $y$, where $y=y_{i}$ for which $p\left(y_{i} \mid x^{\prime}\right)$ was maximized, i.e. $y=y_{i}$, where $\max _{i}\left\{p\left(y_{i} \mid x^{\prime}\right)\right\}$.

Step 6: Considering the TS has the sequences already classified, the True Positive, True Negative, False Positive and False Negative values were obtained and the predicting metrics Accuracy, Precision, Recall and F-measure were computed.

Algorithm 1 - Learning Stage used as training set the sequences from the 58 patients described in Section 2.1, which were obtained as a result of the time series conversions to SAX. Step 1 created the learning set with the sequences and its classifications i.e. 1 and 0 .

Step 2 created the pairdataset, $P S$, as the pair of sequences used as an input to learn the transducer. To create the pairdataset, for each sequence $\mathrm{x}$ the 1-Nearest Neighbor $(N N)$ with the classic edit distance was computed, the pairs $(x, N N(x))$ were formed, both from the learning set.

The stochastic edit costs are obtained by learning the conditional finite-state transducer in Step 3. The recursive forward and backward algorithms were implemented to compute the probabilities $p(y \mid x)$, where $x, y$ were the input data from the pairdataset [13]. Then, the expectation-maximization algorithm was used to optimize the parameters [14]. The expectation algorithm dealt with the problem of obtaining the matrix with the expected values, while maximization computed the current edit costs. These algorithms were repeated until the expected precision was reached (see [7] for more details about the algorithm).

In the Validation Stage, the goal was to test the learned transducer with the edit cost probabilities on a real data set. This dataset consisted in 10 patients and included the sequences and their classifications (more details of the test set were also described in Section 2.1). Then, for each sequence in the test set, the 1-Nearest-Neighbor was used along with the learned stochastic edit distances to classify the sequence as 0 or 1 based on the training set Step $\mathbf{4}$ and 5 .

As the sequences in the test set were already classified, prediction metrics, i.e. Accuracy, Precision, Recall and F-measure, were computed to compare the results of the classifier under test with trusted external judgments. True Positive, True Negative, False Positive and False Negative values were used to compute the metrics, where the terms positive and negative refer to the classifier's prediction and the terms true and false refer to whether that prediction corresponds to the external judgment (Step 5).

\section{Results}

\subsection{Evaluation approach design}

Several experiments are developed in order to obtain the most accurate model that evaluates univariate analysis on the highfrequency data. Implemented Learning and Validations Stages described in Algorithm 1 are repeated for different parameter values. These parameters were changed during the SAX conversion data. The idea behind these experiments is to establish 
the parameters that better adjust the stochastic finitestate transducer model to predict hypotension scenario within an hour.

These parameters, i.e. WindowSize, Symbols and Alphabet, are described in detail in Section 2.1. Nine different experiments are developed. In all cases, a WindowSize $=120$ is used, because expert medical professionals felt that examining two hours of data was the minimal amount of data to capture a stark decline in a patient's condition (an episode of acute hypotension). Parameters Symbols and Alphabet are changed by $[6,12,24]$ and $[3,4,5]$, respectively. The prediction metrics described in Algorithm 1 Step 5, i.e. Accuracy, Precision, Recall and F-measure values are then computed, using the following formulas:

$$
\begin{aligned}
& \text { Accuracy }=\frac{\text { truePos }+ \text { trueNegs }}{\# \text { instansces }} \\
& \text { Precision }=\frac{(\text { truePos })}{\text { truePos }+ \text { falsePos }} \\
& \text { Recall }=\frac{(\text { truePos })}{\text { truePos }+ \text { falseNegs }} \\
& F-\text { Measure }=\frac{2 * \text { Prec } * \text { Recall }}{\text { Prec. }+ \text { Recall }}
\end{aligned}
$$

\subsection{Results by adjusted parameters}

Table 1 describes the results of the experiments. As can be seen, parameters set for Exp. \#1 and \#2 are not even able to produce some of the validation metrics. That is because the data do not provide enough information to correctly learn the probability cost by the stochastic finite-state transducer. The model works better for larger Symbols parameter sizes (Exp. \#7, \#8, \#9). The symbols parameter determines the granularity of the measurement meaning that in a WindowSize of 120 and Symbols value of 24 , each symbol represents 5 min (120/24). The Alphabet value of 3 separates the normalized values into three symbolic representations based on whether the value falls into the low, average or high values in a Gaussian distribution. The best results are obtained when the parameters are set as Symbols $=24$ and Alphabet $=3($ Exp. \#7), where all the validation metrics are greater than 0.8 (i.e. Accuracy $=0: 85$, Precision $=0: 82$, Recall $=0: 87$ and F-Measure $=0: 86$ ).

\subsection{Discussion and related works}

Based on the validation metrics in Exp. \#7, our model makes a good prediction of the patient outcomes. Other results have been previously developed to predict patient status using time series data $[6,15]$. Ordoñez et al. [6] use natural language processing and text mining techniques to predict patient outcomes. We use their study [6] as a baseline to compare with

\begin{tabular}{|c|c|c|c|c|}
\hline \multirow{2}{*}{$\begin{array}{l}\text { Exp. } \\
\#\end{array}$} & \multicolumn{3}{|l|}{ Parameters } & \multirow[t]{2}{*}{ Results } \\
\hline & Window Size & Symbols & Alphabet & \\
\hline 1 & 120 & 6 & 3 & $\begin{array}{l}\text { Accuracy }=0.50 \\
\text { Precision }=0.0 \\
\text { Recall = NaN } \\
\text { F-Measure }=\mathrm{NaN}\end{array}$ \\
\hline 2 & 120 & 6 & 4 & $\begin{array}{l}\text { Accuracy }=0.5 \\
\text { Precision }=0.0 \\
\text { Recall }=\mathrm{NaN} \\
\text { F-Measure = NaN }\end{array}$ \\
\hline 3 & 120 & 6 & 5 & $\begin{array}{l}\text { Accuracy }=0.51 \\
\text { Precision }=0.23 \\
\text { Recall }=0.5 \\
\text { F-Measure }=0.31\end{array}$ \\
\hline 4 & 120 & 12 & 3 & $\begin{array}{l}\text { Accuracy }=0.76 \\
\text { Precision }=0.68 \\
\text { Recall }=0.78 \\
\text { F-Measure }=0.73\end{array}$ \\
\hline 5 & 120 & 12 & 4 & $\begin{array}{l}\text { Accuracy }=0.35 \\
\text { Precision }=0.28 \\
\text { Recall }=0.25 \\
\text { F-Measure }=0.26\end{array}$ \\
\hline 6 & 120 & 12 & 5 & $\begin{array}{l}\text { Accuracy }=0.68 \\
\text { Precision }=0.48 \\
\text { Recall }=0.69 \\
\text { F-Measure }=0.56\end{array}$ \\
\hline 7 & 120 & 24 & 3 & $\begin{array}{l}\text { Accuracy }=0.85 \\
\text { Precision }=0.82 \\
\text { Recall }=0.87 \\
\text { F-Measure }=0.85\end{array}$ \\
\hline 8 & 120 & 24 & 4 & $\begin{array}{l}\text { Accuracy }=0.69 \\
\text { Precision }=0.78 \\
\text { Recall }=0.69 \\
\text { F-Measure }=0.73\end{array}$ \\
\hline 9 & 120 & 24 & 5 & $\begin{array}{l}\text { Accuracy }=0.67 \\
\text { Precision }=0.81 \\
\text { Recall }=0.57 \\
\text { F-Measure }=0.67\end{array}$ \\
\hline
\end{tabular}

Table 1 Results of the validation metrics by changing the parameters of the data conversion when stochastic finite-state transducer model is used to determine the stochastic edit distance costs

our method. They also compute the same prediction metrics (i.e. Accuracy, Precision, Recall and F-measure) to validate their results. Therefore, we compared the best values for each of the prediction metrics in their study and compared them with our results.

As can be seen in Table 2, our results are better than the previous for most of the metrics. Only the Recall metric gives 
Table 2 Metric Comparison: baseline vs current work

\begin{tabular}{lll}
\hline & Ordoñez et al. [5] & Current Work \\
\hline Accuracy & $0.81^{\mathrm{a}}$ & 0.85 \\
Precision & $0.79^{\mathrm{b}}$ & 0.82 \\
Recall & $0.96^{\mathrm{c}}$ & 0.87 \\
F-measure & $0.84^{\mathrm{d}}$ & 0.85 \\
\hline
\end{tabular}

${ }^{\mathrm{a}}$ Most accurate value from Multivariate Piecewise Dynamic Time Warping

${ }^{\mathrm{b}}$ Most precise value from Multivariate Stacked Bags of Patterns

${ }^{\mathrm{c}}$ Highest recall value from Multivariate Piecewise Dynamic Time Warping

${ }^{\mathrm{d}}$ Highest F-measure value from Multivariate Piecewise Dynamic Time Warping

better value when it is compared with our current research. It is also important to consider that we only use one univariate variable, Heart Rate, to predict hypotension while [6] uses a multivariate representation using Heart Rate and Mean Arterial Pressure.

We had to reduce the size of the time series because of computational limitations to only 1020 characters. In [6], the multivariate representations with two variables outperformed the univariate representations. In this paper, even with more dimensionality reduction, the univariate representation outperformed the work of [6]. Although we have obtained good results, we still consider that Algorithm 1 implementation can be improved to handle larger sequences and multivariate representations to obtain more precise results.

\section{Conclusion and future works}

In this research, we proposed a method to predict patient outcomes in Intensive Care Units which used probabilities of edit distance costs learned by stochastic finite-state transducer models. Time series data were converted to sequence representation to be used as a model input. Several experiments were developed by changing the parameters during the conversion process. We obtained good results based on the computed prediction metrics. When we compare with previous works, our proposal improved Accuracy, Precision and Fmeasure metric values.

In future work, other implementations of the algorithm in parallel will be used to increase the sequence length and improve efficiency. We would also like to create a multivariate representation of the algorithm. Additionally, other approaches using finite-state transducers can be used to improve prediction. For example, rational kernels (kernel based on finite-state transducers) can be combined with kernel methods, such as Support Vector Machine [15].
Acknowledgments This work was partially funded by Natural Sciences and Engineering Research Council of Canada (NSERC) at University of Manitoba, Canada, RCMI grant G12 MD007600 (National Institute on Minority Health and Health Disparities) from the National Institutes of Health at University of Puerto Rico Medical Sciences Campus, and the NIH grant 11882032 from the National Institutes of Health at the University of Puerto Rico Río Piedras Campus.

\section{Compliance with ethical standards}

Conflict of interest The authors declare that there are no conflicts of interest regarding the publication of this manuscript.

Open Access This article is distributed under the terms of the Creative Commons Attribution 4.0 International License (http:// creativecommons.org/licenses/by/4.0/), which permits unrestricted use, distribution, and reproduction in any medium, provided you give appropriate credit to the original author(s) and the source, provide a link to the Creative Commons license, and indicate if changes were made.

\section{References}

1. Oster J, Clifford GD. Signal quality indices for state space electrophysiological signal processing and vice versa. In: Chen $\mathrm{Z}$, editor. Advanced state space methods for neural and clinical data. Cambridge University Press; 2015. p. 345-366.

2. Erol, YB Phadte R, Sidhu HS, Asselstine C, Phillips D, Manley G, Russell S. Model based probabilistic inference for intensive care medicine. In: Meaningful use of complex medical data, 2015. http://mucmd.org/conference-2015.html Accessed: 30 Aug 2016.

3. Batal I, Fradkin D, Harrison J, Moerchen F, Hauskrecht M. Mining recent temporal patterns for event detection in multivariate time series data. In: Proceedings of the 18th ACM SIGKDD International Conference on Knowledge Discovery and Data Mining, KDD '12, New York, NY, USA. 2012. doi:10.1145 /2339530.2339578, p. 280-288.

4. Wang Z, Oates T. Pooling sax-bop approaches with boosting to classify multivariate synchronous physiological time series data. In: Proceedings of the Twenty-Eighth International Florida Artificial Intelligence Research Society Conference, FLAIRS 2015, Hollywood, Florida, 2015. http://www.aaai.org/ocs/index. php/FLAIRS/FLAIRS15/paper/view/10384, p. 335-341.

5. Wang Z, Oates T. Imaging time-series to improve classification and imputation. CoRR, abs/1506.00327, 2015. http://arxiv. org/abs/1506.00327 Accessed: 30 Aug 2016.

6. Ordoñez P, Armstrong T, Oates T, Fackler J, Lehman UC. Multivariate methods for classifying physiological data. In: Proceedings of SIAM International Conference on Data Mining, Workshop on Data Mining Medicine and HealthCare (DMMH 2013). 2013. p. 37.

7. Oncina J, Sebban M. Learning stochastic edit distance: application in handwritten character recognition. Pattern Recogn. 2006;39(9): 1575-87.

8. Moody GB, Lehman LH. Predicting acute hypotensive episodes: the 10th annual physionet/computers in cardiology challenge. Comput Cardiol 2009; 541-544.

9. LCP. Predicting acute hypotensive episodes. 2009. http://physionet. org/challenge/2009/. Accessed: 20 May 2016.

10. Lin J, Keogh E, Wei L, Lonardi S. Experiencing sax: a novel symbolic representation of time series. Data Min Knowl Disc, 2007. ISSN 1573-756X. 15(2):107-144. doi:10.1007/s10618-007-0064-z. 
11. De la Higuera C. Grammatical inference: learning automata and grammars: Cambridge University Press; 2010.

12. Egho E, Raïssi C, Calders T, Jay N, Napoli A. On measuring similarity for sequences of itemsets. Data Min Knowl Disc. 2015;29(3): 732-64.

13. Govindaraju SG, Dill DL. Verification by approximate forward and backward reachability. In: Computer-Aided Design. ICCAD 98. Digest of Technical Papers. 1998 IEEE/ACM International Conference on; 1998. p. 366-370.
14. Lawrence CL, Reilly AA. An expectation maximization (EM) algorithm for the identification and characterization of common sites in unaligned biopolymer sequences. Proteins: Structure, Function, and Bioinformatics. 1990;7(1):41-51.

15. Lin J, Li Y. Finding structural similarity in time series data using bag-of-patterns representation. In: Proceedings of the $21 \mathrm{st}$ International Conference on Scientific and Statistical Database Management, SSDBM 2009, Berlin, Heidelberg: Springer-Verlag, ISBN 978-3-642-02278-4, 2009. pp. 461-477. 